\title{
Integrated approach to energy harvester mixed technology modelling and performance optimisation
}

\author{
Leran Wang, Tom J. Kazmierski, Bashir M. Al-Hashimi, \\ Steve P. Beeby and Russel N. Torah \\ School of Electronics and Computer Science \\ University of Southampton, UK \\ $\{1 \mathrm{lw} 04 \mathrm{r}, \mathrm{tjk}, \mathrm{bmah}, \mathrm{spb}, \mathrm{rnt}\} @$ ecs.soton.ac.uk
}

\begin{abstract}
This paper presents an integrated approach to energy harvester modelling and performance optimisation where the complete mixed physical-domain energy harvester system (micro generator, voltage booster, storage element and load) can be modelled and optimised in a systematic manner using one simulation platform. We developed an accurate HDL model for the energy harvester and demonstrated its accuracy by validating it experimentally and comparing it with recently reported models. To address the performance loss due to the close mechanical-electrical interaction that takes place in energy harvesters, we proposed a holistic methodology to the energy harvester optimisation based on the HDL model. The effectiveness of employing such an approach has been demonstrated by showing that it is possible to improve vibration-based energy harvester efficiency (energy delivered to load/harvested energy) by $30 \%$ through optimising the micro-generator size and the voltage booster circuit components.
\end{abstract}

\section{Introduction}

Pervasive computing (integration of computation into our daily lives through sensing systems to enhance our activities without being intrusive), is widely believed to represent the next major market for the electronics industry. One of the key challenges in pervasive computing research is energy harvesting (conversion of ambient energy captured from the environment into electrical energy), where the focus is to develop energy harvesters to power electronic devices, particularly in wearable and embedded sensors [7], where batteries are cumbersome or impractical.

There are considerable and continuing research efforts world-wide to support the energy harvesting paradigm and self-powered electronics. Majority of the reported research in energy harvesting has been on improving the efficiency of the energy harvesters through the design and fabrication of novel micro-generators, materials and devices $[2,4]$. Because of the output power of energy harvesters is highly variable and unpredictable, recently Amirtharajah et al. [1] proposed digital circuits that employ serial computation and distributed arithmetic as a way of dealing with the variability of the available energy. Also, in [10], it was reported through the use of dedicated sub-threshold logic it is possible to implement an FFT processor that operate in practice with supply voltage as low as $180 \mathrm{mV}$, suitable for energy harvesters.

An energy harvester is a system consisting of several components from different physical domains including mechanical, magnetic and electrical as well as the external circuits which regulate and store the generated energy. To design highly efficient energy harvesters, we believe that it is crucial to consider the various parts of an energy harvester in the context of a complete system, or else the gain at one part may come at the price of efficiency loss else where, rending the energy harvester much less efficient than before. Furthermore, the close mechanical-electrical interaction that takes place in energy harvesters, often lead to significant performance loss when the various parts of the energy harvesters are combined. However, there has been little reported research on systematic modelling and optimisation of energy harvester so the aim of this paper is to propose such an approach. Some reported circuit designs treat the micro-generator as an ideal voltage source [11] but we show that modelling the micro generators using ideal voltage source correlates poorly with practice. Other reported designs use a simple linear equivalent circuit model [1] but we also show that simulation results from such a model are not accurate. Based on this motivation, we developed a mixed physical-domain behavioural model. Mixedtechnology HDL modelling itself is not new, but what is new here is that HDL provides accurate modelling tech- 
nique for energy harvester. Also, enhanced performance of energy harvester is achieved through the use of HDLbased optimisation. Several HDLs that support multiple domain system modelling and simulation are available, such as VHDL-AMS, Verilog-AMS and SystemC-A. In this paper VHDL-AMS [3] has been chosen as the modelling language.

\section{Energy harvester modelling}

Vibration-based electromagnetic energy harvesters are proving to be successful when applied in real-world applications [8] and therefore are used in this paper as proof-ofconcept for the proposed modelling and performance optimisation integrated approach. It should be noted that $2 \mathrm{~mW}$ can be generated from a vibration magnitude of $25 \mathrm{mg}_{R M S}$ at $17.2 \mathrm{~Hz}$ [8].

An energy harvester has normally three main components: the micro generator which converts ambient environment energy into electrical energy, the voltage booster which pumps up and regulates the generated voltage, and the storage element (Figure 1).

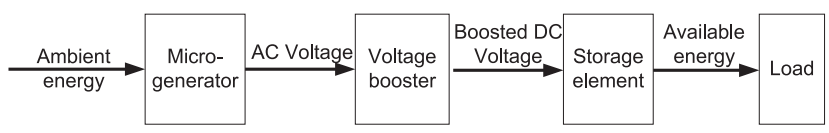

Figure 1. Block diagram of an energy harvester.

Reported modelling approaches aim to replace the micro generator of an energy harvester with either an ideal voltage source (Figure 2 (a)) or an equivalent circuit model (Figure 2 (b)) when designing the voltage booster. However, as will be shown in this paper, neither of these approaches is suitable for accurate voltage booster design.

The proposed approach in this paper uses VHDL-AMS to describe the micro generator as a series of analytical equations (Figure 2 (c)), which includes mechanical, magnetic and electrical behaviours of the micro generator. Through out the paper, comparisons have been made between different modelling approaches and we show that our HDL-based model is more accurate than the circuit models.

The case study presented in this paper uses a vibrationbased electromagnetic micro generator [9] as an example. The design is based on a cantilever structure. The coil is fixed to the base and four magnets, which are located on both sides of the coil, form the proof mass (Figure 3 (a)). This structure can be modelled as a second-order spring-damping system, which has been widely used [2], and whose dynamics is:

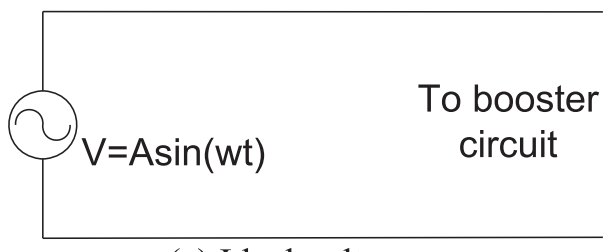

(a) Ideal voltage source

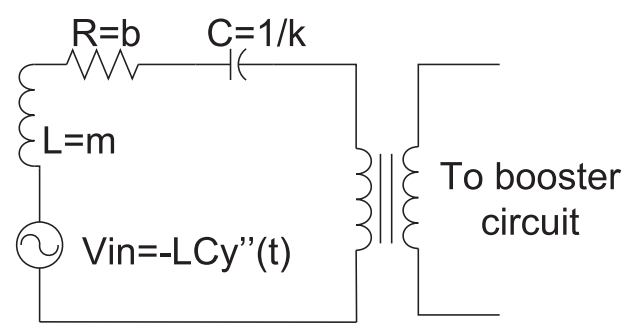

(b) Equivalent circuit model

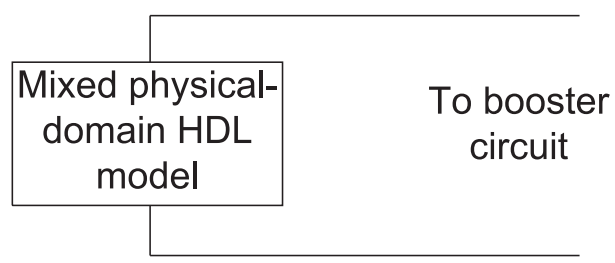

(c) Proposed HDL model

Figure 2. Micro generator models.

$$
\left.m z \ddot{(t)}+c_{p} z(t)+k_{s} z(t)+F_{e m}=-m y \ddot{(t}\right)
$$

where $m$ is the proof mass, $z(t)$ is the relative displacement between the mass and the base, $c_{p}$ is the parasitic damping factor, $k_{s}$ is the spring stiffness, $y(t)$ is the displacement of the base and $F_{e m}$ is the electromagnetic force.

The electromagnetic voltage generated in the coil is given:

$$
v_{\text {em }}=\Phi(z) * z \dot{(t)}
$$

where $\Phi(z)$ is the magnetic flux through the coil.

Although our HDL model is based on analytical equations, it can capture practical size and shape of the actual device. The coil in the actual micro generator consists of $N$ turns and has an inner diameter $r$ and outer diameter $R$. Each of the four opposite magnets are of height $H$ (Figure 3 (a)). So the actual magnetic flux through the coil is a piecewise non-linear function of the relative displacement $z(t)$ : $\Phi=f\{z(t)\}$.

When the relative displacement is small $|z(t)|<r$ (Figure $3(b))$ :

$$
\Phi=\left(\sqrt{R^{2}-z^{2}(t)}+\sqrt{r^{2}-z^{2}(t)}\right) * 2 * B * N
$$




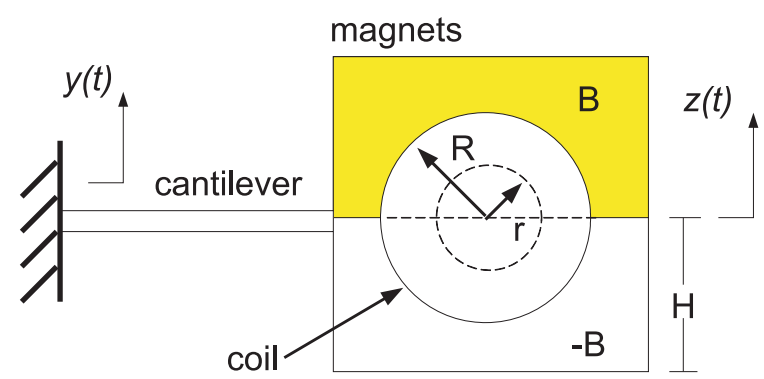

(a) Initial position $z(t)=0$

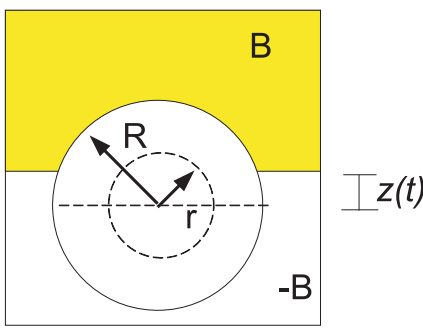

(b) Small displacement $|z(t)|<r$

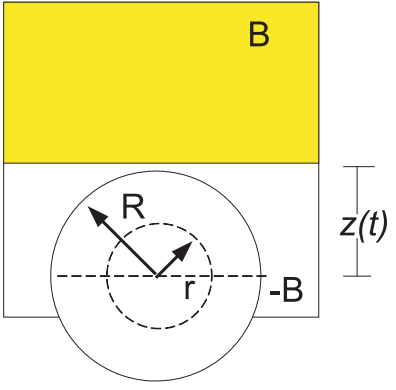

(c) Large displacement $H-r<|z(t)|<H$

Figure 3. Relative displacement between the coil and magnets in the micro generator.

When the relative displacement is large $H-r<|z(t)|<$ $H$ (Figure 3 (c)):

$\Phi=-\left(\sqrt{R^{2}-(H-|z(t)|)^{2}}+\sqrt{r^{2}-(H-|z(t)|)^{2}}\right) * B * N$

There are 5 other sections of the piecewise function which have been implemented in the VHDL-AMS model but are omitted here due to space limitation.

The output voltage is defined by:

$$
v(t)=v_{e m}-R_{c} * i(t)-L_{c} * i \dot{(t)}
$$

where $R_{c}$ and $L_{c}$ are the resistance and inductance of the coil respectively and $i(t)$ is the current through the coil.

Finally, the electromagnetic force is calculated as:

$$
F_{\text {em }}=\Phi(z) * i(t)
$$

There are a number of circuit topologies that can be used as a voltage booster. In this paper we model the voltage booster using VHDL-AMS which describes the circuit behaviour by differential equations.

In the case of storage element, a super capacitor has been modelled by:

$$
C \frac{d\left(V_{C}+V_{L O S T}\right)}{d t}=-I_{C}
$$

where $C$ is the capacitor value, $V_{C}$ and $I_{C}$ are capacitor voltage and current respectively and $V_{L O S T}$ represents the effect of leakage loss.

\section{Models comparison}

In this section we compare the accuracy of different micro generator models with the same voltage booster. Voltage multipliers (VMs) are often used to rectify and boost up AC voltages and thus can act as the voltage booster in an energy harvester. Figure 4 shows a 6-stage Villard voltage multiplier [11], which has been used in the following comparison.

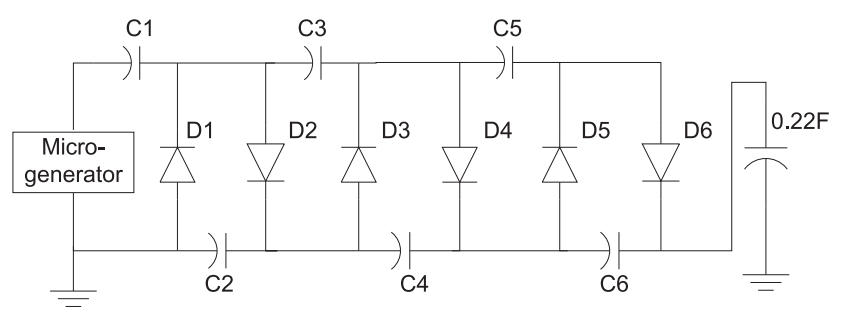

Figure 4. Villard voltage multiplier as the voltage booster.

All the comparisons presented are based on the charging of a $0.22 \mathrm{~F}$ super capacitor. Because the VHDL-AMS simulator used here, SystemVision from Mentor Graphics [5], has a maximum simulation time of 150 minutes, only simulation results in this range have been obtained. Figure 5 shows the simulated charging waveform of the capacitor using different micro-generator models including the proposed HDL model. Also shown is the capacitor charging waveform obtained experimentally. The experiment was setup as the micro-generator sitting on a vibration generator, which produces constant mechanical vibrations (Figure $6)$.

As it can be seen, there is poor correlation between the energy harvester experimental measurements and that of models based on ideal voltage source or equivalent circuit. The reason why the model with ideal voltage source (Figure 2 (a)) fails is that in an energy harvester the voltage booster can greatly affect the behaviour of the micro generator but an ideal voltage source always produces constant output. The crucial mechanical-electrical interaction is missing in the model. As for the equivalent circuit model (Figure 2 


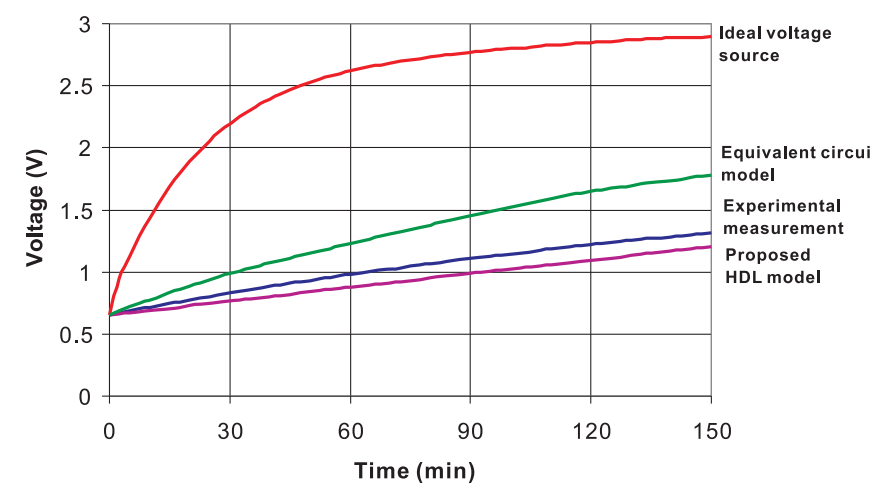

Figure 5. Different energy harvester models compared with experimental measurements.

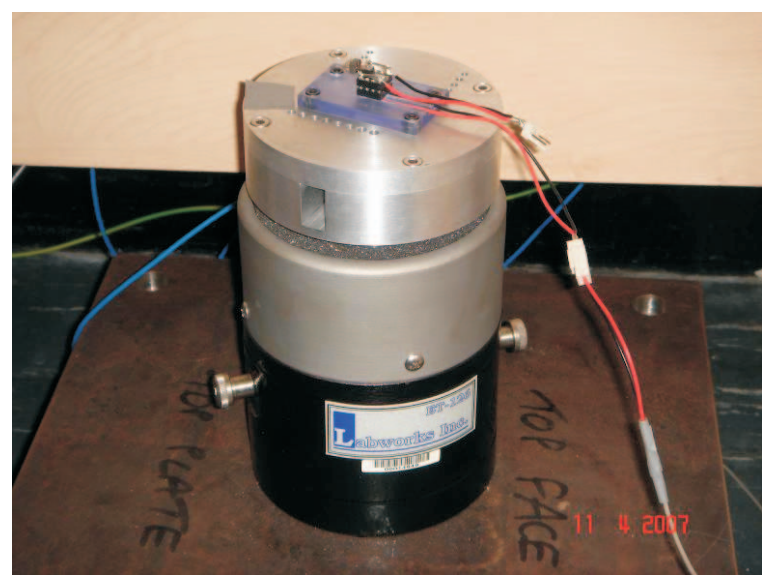

Figure 6. Experimental measurement setup.

(b)), this is because in [1], the equivalent circuit model of the micro generator links mechanical mass $(m)$, $\operatorname{spring}(k)$ and damper $(b)$ to electrical inductor $(L)$, capacitor $(C)$ and resistor $(R)$ by:

$$
L=m, \quad C=1 / k, \quad R=b
$$

which is over simplification and does not capture accurately the internal operation of the energy harvester.

In the case of the proposed energy harvester HDL model, there is a good correlation between the experimental and simulation results. The reason why the HDL-based model correlates well with practice is that it can incorporate the actual shape and size of various components into the micro generator model by using analytical equations. Here the non-linear dependence of the micro generator's output voltage on the input displacement described in Section 2 can be accurately captured by the HDL model. Simulation results of the equivalent circuit model and HDL model are com- pared with the experimental measurement in Figure 7. As can be seen from the waveforms, when excited by a sine wave stimulus, the equivalent circuit model still generates sine wave output. But the HDL model can capture the situations when the coil and magnets are moving apart, which is what happens in practice and leads to non-sine wave output.

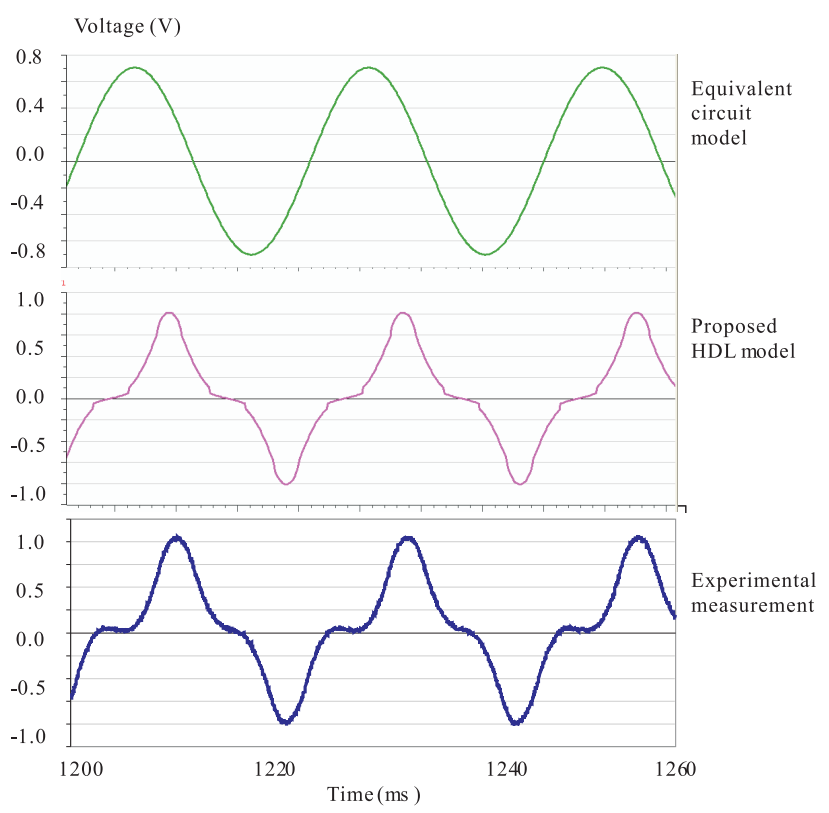

Figure 7. Nonlinear output from micro generator correctly reflected by proposed model.

\section{Performance optimisation}

The close mechanical-electrical interaction (microgenerator and voltage booster) that takes place in energy harvesters, often lead to significant performance loss when the various parts of the energy harvesters are combined. Here the loss expressed in terms of energy harvesting efficiency:

$$
\eta_{\text {Loss }}=\frac{E_{\text {Harvested }}-E_{\text {Delivered }}}{E_{\text {Harvested }}}
$$

Therefore, to address the performance loss, we propose an integrated approach to the energy harvester modelling and performance optimisation (Figure 8). In our approach, not only the energy harvester model but also the optimisation algorithm is implemented in a single VHDL-AMS testbench. The parameters used for the optimisation are from both the micro generator and the voltage booster. The optimisation object is to increase the charging rate of the super capacitor. The optimisation algorithm generates design parameters to the model and obtains the charging rate through 
simulation. The optimisation loop runs continuously until the design parameters reach an optimum.

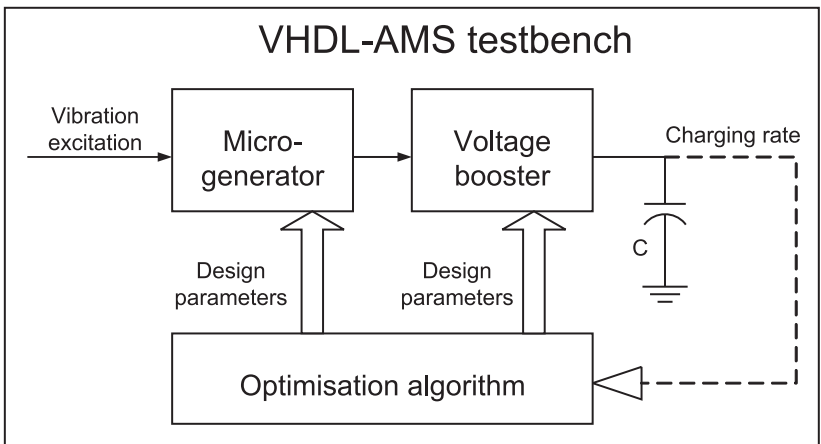

\section{Figure 8. Integrated performance optimisa- tion in VHDL-AMS testbench.}

A super capacitor of $0.22 \mathrm{~F}$ has been used in the performance optimisation experiment. The micro-generator parameters that can be optimised are related to the coil size, i.e the number of turns $(N)$, the internal resistance $\left(R_{c}\right)$ and the outer diameter $(R)$. Because other components such as the magnets and cantilever determine the resonant frequency of the micro-generator and thus should be based on application requirements. The circuit parameters of voltage booster have also been included and the entire energy harvester is optimised as an integrated model. For proof of concept, we employed a genetic algorithm (GA) [6] to optimise the energy harvester with a voltage transformer booster. Other optimisation algorithms may also be applied based on the proposed integrated model.

\section{Integrated approach simulation results}

To validate the effectiveness of the proposed approach to reduce the performance loss in energy harvester (and improve energy harvesting efficiency) due to the interaction between the mechanical and electrical parts, we have carried the following simulation. The vibration-based microgenerator is based on the cantilever structure and has been designed separately from the remaining parts of the energy harvester (Figure 1). The structure coil parameters are given in Table 1. The voltage booster circuit (Figure 9) has also been designed independently from the rest of the energy harvester and its parameters are given in Table 1. Note we have used this booster circuit in stead of the circuit in Figure 4 since it has better performance. This energy harvester design is referred to as "un-optimised" since the micro-generator and the voltage booster have been designed separately.

The implemented GA has a population size of 100 chromosomes. Each chromosome has 7 parameters (3 from

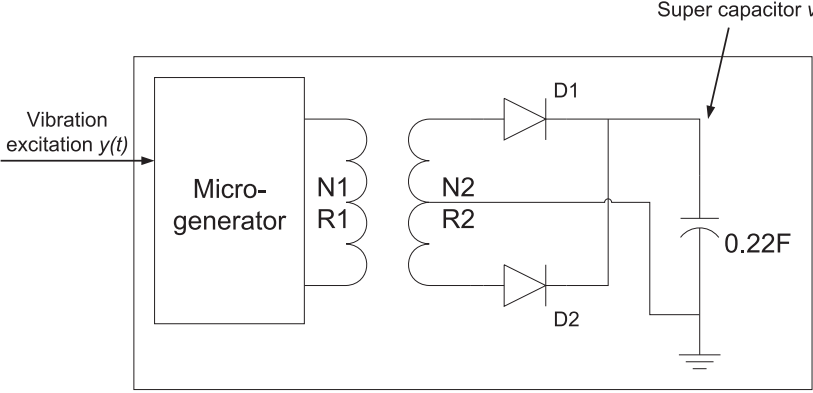

Figure 9. Transformer based voltage booster.

\begin{tabular}{|c|c|c|}
\hline \multicolumn{3}{|c|}{ Micro-generator } \\
\hline Outer radiu & $f$ coil $(R)$ & $1.2 \mathrm{~mm}$ \\
\hline Coil tu & $S(N)$ & 2300 \\
\hline Internal res & ance $\left(R_{c}\right)$ & $1600 \Omega$ \\
\hline \multicolumn{3}{|c|}{ Voltage transformer } \\
\hline & Resistance $(\Omega)$ & No. of turns \\
\hline Primary winding & 400 & 2000 \\
\hline Secondary winding & 1000 & 5000 \\
\hline
\end{tabular}

Table 1. Parameters of un-optimised energy harvester.

the micro-generator and 4 from the voltage booster). The crossover and mutation rate are 0.8 and 0.02 respectively. The chromosome's fitness is updated every $50 \mathrm{~ms}$. After simulating the testbench for 100 seconds, which corresponds to 2000 generations in the GA optimization, the gene values converge to an optimum. The simulation CPU time is 17 hours on a Pentium 4 PC. Note that the long simulation time is not due to the GA employed, because majority of the simulation time was dedicated to the simulation of 100 chromosomes for every GA generation. For indication, CPU time of $10 \mathrm{GA}$ generations $(500 \mathrm{~ms})$ is 181 seconds and simulating 100 chromosomes for $500 \mathrm{~ms}$ alone (without GA) takes 177 seconds, which means that GA accounts for less that $3 \%$ of the CPU time. In particular, the energy harvester has a large storing capacitor of $0.22 \mathrm{~F}$ and even for one chromosome, simulating the voltage across it with time step of $0.1 \mathrm{~ms}$ for 100 seconds takes almost $10 \mathrm{~min}$ utes. Although optimisation speed is not a main concern here, VHDL-AMS is known to be slow for pure mathematical computations and may not be suitable for some more complicated optimisation algorithms. Here we use VHDLAMS to implement the GA because this approach yields an integrated optimisation system wholly implemented in the hardware description language and it allows automatic optimisation using a single platform.

Applying the proposed modelling and performance opti- 
misation, Table 2 gives the new micro-generator and voltage booster parameters which are referred to as "optimised" design. The impact of these values on improving the charging of the super is shown in Figure 10. As can be seen from the simulation results, in 150 minutes the un-optimised energy harvester charges the super capacitor to $1.5 \mathrm{~V}$ and the optimised energy harvester reaches $1.95 \mathrm{~V}$, which represents a $30 \%$ improvement.

Micro-generator

\begin{tabular}{|c|c|c|}
\hline Outer radil & of coil $(R)$ & $1.1 \mathrm{~mm}$ \\
\hline Coil t & $\mathrm{s}(N)$ & 2100 \\
\hline Internal re & ance $\left(R_{c}\right)$ & $1400 \Omega$ \\
\hline \multicolumn{3}{|c|}{ Voltage transformer } \\
\hline & $\operatorname{Resistance}(\Omega)$ & No. of turns \\
\hline Primary winding & 340 & 1900 \\
\hline Secondary winding & 690 & 3800 \\
\hline
\end{tabular}

\section{Table 2. Parameters of optimised energy har- vester.}

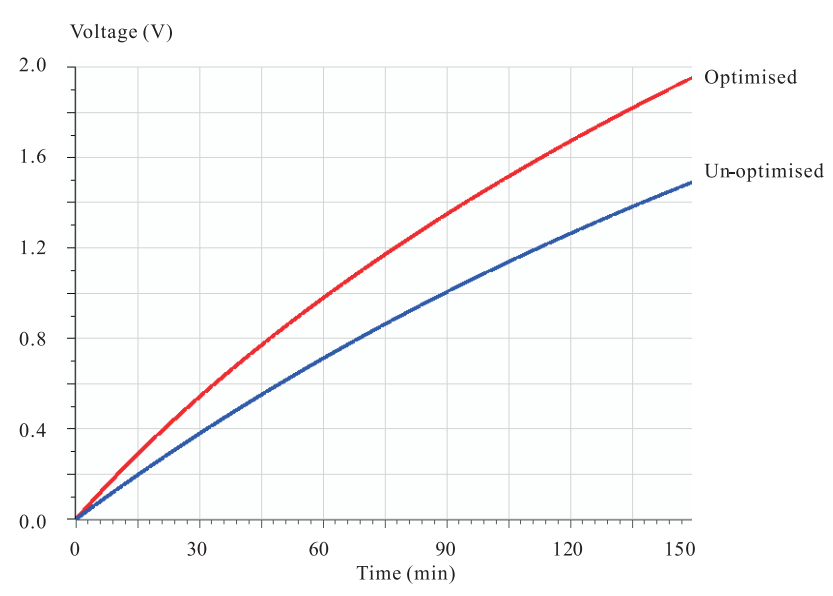

Figure 10. Simulation waveforms of super capacitor charging by different energy harvester models.

\section{Conclusion}

It is likely that energy harvesters will have a key role to play in providing the energy needed to power up modern electronics present in several emerging applications. To maximize the energy harvester performance, we believe that various parts of energy harvesters (mechanical and electrical) need to be optimised in a holistic and integrated approach. This paper presented such an approach to the modelling and optimisation of energy harvesters through the em- ployment of a mixed-technology HDL. We have shown that the existing energy harvester modelling approaches are inadequate and we have demonstrated the effectiveness of the proposed approach through the case study of an electromagnetic energy harvester. It has been shown that through performance optimisation it is possible to achieve a $30 \%$ improvement in the super capacitor charge rate.

\section{ACKNOWLEDGMENTS}

The authors would like to thank the Engineering and Physical Sciences Research Council (EPSRC-UK) for funding this work in part under grant number GR/S95770/01.

\section{References}

[1] R. Amirtharajah, J. Wenck, J. Collier, J. Siebert, and B. Zhou. Circuits for energy harvesting sensor signal processing. In Design Automation Conference, 2006 43rd ACM/IEEE, pages 639-644, 24-28 July 2006.

[2] S. P. Beeby, M. J. Tudor, and N. M. White. Energy harvesting vibration sources for microsystems applications. Measurement science and technology, 17(12):R175-R195, 2006.

[3] E. Christen and K. Bakalar. VHDL-AMS-a hardware description language for analog and mixed-signal applications. Circuits and Systems II: Analog and Digital Signal Processing, IEEE Transactions on, 46(10):1263-1272, Oct. 1999.

[4] K. Makihara, J. Onoda, and T. Miyakawa. Low energy dissipation electric circuit for energy harvesting. Smart materials and structures, 15(5):1493-1498, 2006.

[5] Mentor Graphics Corporation. SystemVision User's Manual. Version 3.2, Release 2004.3. July 2004.

[6] M. Mitchell. An Introduction to Genetic Algorithms. the MIT Press, Cambridge, Massachusetts, 1996.

[7] J. A. Paradiso and T. Starner. Energy scavenging for mobile and wireless electronics. Pervasive Computing, IEEE, 4(1):18-27, 2005.

[8] Perpetuum Ltd. http://www.perpetuum.co.uk/.

[9] R. Torah, S. P. Beeby, M. J. Tudor, T. O’Donnell, and S. Roy. Development of a cantilever beam generator employing vibration energy harvesting. In Proceedings of The 6th Int. Workshop on Micro and Nanotechnology for Power Generation and Energy Conversion Applications, pages 181-184, 2006.

[10] A. Wang and A. Chandrakasan. A 180-mv subthreshold fet processor using a minimum energy design methodology. IEEE Journal of Solid State Circuits, 40(1):310-319, 2005.

[11] H. Yan, J. Macias Montero, A. Akhnoukh, L. de Vreede, and J. Burghartz. An integration scheme for rf power harvesting. In Proc. STW Annual Workshop on Semiconductor Advances for Future Electronics and Sensors, pages 64-66, 17-18 November 2005. 